\title{
Physical Characteristics of Stream Subbasins in the North Fork Crow-Crow River Basin, South-Central Minnesota
}

\author{
By Christopher A. Sanocki
}

\begin{abstract}
Data that describe the physical characteristics of stream subbasins upstream from selected sites on streams in the North Fork Crow-Crow River Basin, located in south-central Minnesota are presented in this report. The physical characteristics are the drainage area of the subbasin, the percentage area of the subbasin covered only by lakes, the percentage area of the subbasin covered by both lakes and wetlands, the main-channel length, and the main-channel slope. Stream sites include outlets of subbasins of at least 5 square miles, and locations of U.S. Geological Survey low-flow, high-flow, and continuous-record gaging stations.
\end{abstract}

\section{Introduction}

This is the 14th report in a series detailing subbasin characteristics of streams in Minnesota and adjacent states. The North Fork Crow-Crow River Basin drains an area of 1,480 square miles and is represented by hydrologic accounting unit 07010204 (U.S. Geological Survey, 1974). The North Fork Crow-Crow River Basin includes parts of Carver, Hennepin, Kandiyohi, Meeker, Mc Leod, Pope, Stearns, and Wright Counties in southcentral Minnesota.

Selected data for sites on streams at outlets of subbasins larger than about 5 square miles; at locations of U.S. Geological Survey (USGS) low-flow, high-flow, and continuous-record gaging stations located in the North Fork Crow-Crow River Basin are presented in this report. This report was prepared in cooperation with the Minnesota Department of Transportation.

\section{Acknowledgments}

Brian Schreurs, a graduate student at St Cloud State University, did much of the digitizing and assisted in the preparation of this report. These contributions were essential for the completion of this report.

\section{Methods}

USGS 7-1/2 minute series topographic maps were used as source maps to obtain the areas for the subbasin boundaries, the main-channel length, and the contour elevation points used in this report. Paper copies of the maps were used. Lake and marsh data were obtained from U.S. Fish and Wildlife Service National Wetlands
Inventory Data (U.S. Fish \& Wildlife Service, 1981present). A geographic information system (GIS) was used to define the geographic location and extent of the subbasins, lakes, marshes, main-channels, and elevation points. Data digitized from paper copies were in error by no more than twice the horizontal accuracy of National Mapping Standards of 40 feet (Thompson, 1987, p. 104). All thematic (digitized) data were projected into an Albers Equal-Area projection for storage and analysis.

Subbasin boundaries were delineated on the basis of anthropogenic activities and topographic contours. Anthropogenic activities, such as the installation of storm sewers, the drainage of wetlands, and the diversion of streams, may alter the drainage area of a stream. Data from field inspections and recent drainageditch maps, therefore, were transferred to the topographic maps. The subbasin boundaries were digitized by the Minnesota Department of Natural Resources (DNR), and the USGS, Minnesota using a GIS.

Lake and marsh boundaries were overlaid on the subbasin boundaries to associate each lake and marsh with a subbasin. The total area of lakes and marshes within each subbasin was calculated by the GIS. Total marsh area plus total lake area is defined as storage area.

Main channels were delineated for each subbasin on the 7-1/2 minute topographic maps starting at the outflow of the subbasin and continuing upstream. Whenever the main channel joined with another stream, the stream upstream of the junction that drained the largest area was selected as the main channel. The main 
channel, which represents the watercourse that drains the greatest area, is continuous and is defined as a single trace that passes through marshes, lakes, and midline of rivers and braided streams from the basin outlet to an endpoint in the basin, generally at the basin divide. The main channels were digitized by the Minnesota Department of Transportation, using a computer aided drafting system and transferred to the GIS. Stream extensions that represent a portion of the main channel from the end of the mapped stream (blue line on 7-1/2 minute topographic maps) to an endpoint within the basin, generally at the basin divide, were digitized by USGS Minnesota using a GIS. The main-channel data were overlaid onto the subbasin data to associate each main channel with its subbasin.

Elevation points were digitized at the intersection of topographic contour lines and main channels. The elevation data were digitized using a GIS. The elevation data was overlaid onto the main channel data to associate each elevation data point with a main channel. Two points on the main channel, at 10 percent and at 85 percent of the main channel length from the basin outlet to the drainage divide, were located by the GIS. The elevations of these two points were interpolated from the digitized elevation data. Main-channel slope was calculated by dividing the difference in elevation between these points by the distance along the stream channel between these points.

\section{Physical Characteristics of North Fork Crow-Crow River Subbasins}

Physical characteristics determined for each of the subbasins shown on plate 1 are presented in table 1 . Subbasins are presented in order from headwaters to mouth. The rank of the subbasin stream is shown by indentation; whenever two subbasin streams joined, the stream draining the least cumulative area was assigned a lower rank and indented in the table.

The data for drainage area, and main-channel length are reported using three significant figures or rounded to the nearest one-hundredth of a unit. The data for lake area and storage area are reported using two significant figures or rounded to the nearest one-tenth of a percent. The data for main-channel slope is reported to the nearest one-tenth of a foot per mile.

The following is an explanation of the terms used in table 1:

Subbasin number. A seven digit number based on the Minnesota Common Stream and Watershed Numbering System (Minnesota Department of Natural Resources, 1981). The first two digits are 18 and identify the North
Fork Crow-Crow River Basin. The following three digits are arbitrary and were assigned by the DNR. The last two digits were added by the USGS Minnesota, to identify additional subdivisions to the DNR's watersheds at locations of USGS gaging stations and to identify noncontributing areas.

Stream name. The name of the stream or ditch shown on $7-1 / 2$ minute topographic maps. The relative position of the subbasin above other subbasins, streams, and gaging stations.

Outlet location. The U.S. Public Lands Survey System is used to describe the location where the stream exits the subbasin, down to quarter-quarter section. The description includes quarter-quarter section, section, township, and range.

Drainage area. That area, measured on a horizontal plane, enclosed by a topographic divide, within which direct surface runoff from precipitation normally flows by gravity into a watercourse above a specific point. This may include closed basins and other areas that do not contribute directly to surface runoff.

Lake area. The percentage of the drainage area labeled lacustrine (lakes) on U.S. Fish and Wildlife Service National Wetlands Inventory Data.

Storage area. The percentage of a drainage area labeled lacustrine (lakes) and palustrine (wetlainds)on U.S. Fish and Wildlife Service National Wetlands Inventory Data. Marsh areas shown on plate 1 are from USGS 1:100,000 Digtial Line Graph data 1993.

Main-channel length. The total length of the main channel from the basin outlet to a point within the basin (generally at the basin divide) representing the watercourse that drains the greatest area.

Main-channel slope. The average slope of the watercourse between the points at 10 and at 85 percent of the distance along the main-channel from the basin outlet to the drainage divide.

Stream extension. A representation of the main channel from the end of the mapped stream line (blue line on 7-1/2 minute topographic maps) to an endpoint within the basin, generally at the basin divide. This is done by interpreting topographic relief so the extension of the main channel represents the watercourse draining the greatest area. 


\section{References Cited}

Minnesota Department of Natural Resources, 1981, The common stream and watershed numbering system: Minnesota Department of Natural Resources Stream Inventory and Data Retrieval Systems Report 7002, unpaged.

Thompson, M.M., 1987, Maps for America, 3d edition: U.S. Geological Survey, 265 p.

U.S. Geological Survey, 1974, Hydrologic unit map-1974 State of Minnesota: 1 plate, scale 1:500,000.

U.S. Fish \& Wildlife Service, National Wetlands Inventory Digital Data: Oct. 1981 to present 
Table 1.-Physical characteristic data for the North Fork Crow-Crow River Basin.

[All cities and towns are in Minnesota; --, not determined, Storage area is both lakes and wetlands]

\begin{tabular}{|c|c|c|c|c|c|c|c|c|c|c|c|c|c|}
\hline \multirow[b]{2}{*}{$\begin{array}{c}\text { Basin } \\
\text { number }\end{array}$} & \multirow[b]{2}{*}{ Stream name and location } & \multicolumn{4}{|c|}{ Outlet location } & \multicolumn{3}{|c|}{ By subbasin } & \multicolumn{5}{|c|}{ Cumulative to mouth of basin } \\
\hline & & $\begin{array}{l}\text { Quarter- } \\
\text { quarter } \\
\text { section }\end{array}$ & Section & $\begin{array}{l}\text { Town- } \\
\text { ship }\end{array}$ & Range & $\begin{array}{c}\text { Drainage } \\
\text { area } \\
\text { (square } \\
\text { miles) }\end{array}$ & $\begin{array}{c}\text { Lake } \\
\text { area } \\
\text { (percent } \\
\text { of } \\
\text { subbasin } \\
\text { area) }\end{array}$ & $\begin{array}{c}\text { Storage } \\
\text { area } \\
\text { (percent } \\
\text { of } \\
\text { subbasin } \\
\text { area) }\end{array}$ & $\begin{array}{c}\text { Drainage } \\
\text { area } \\
\text { (square } \\
\text { miles) }\end{array}$ & $\begin{array}{c}\text { Lake } \\
\text { area } \\
\text { (percent } \\
\text { of total } \\
\text { area) }\end{array}$ & $\begin{array}{c}\text { Storage } \\
\text { area } \\
\text { (percent } \\
\text { of total } \\
\text { area) }\end{array}$ & $\begin{array}{c}\text { Main } \\
\text { channel } \\
\text { length } \\
\text { (miles) }\end{array}$ & $\begin{array}{c}\text { Main } \\
\text { channel } \\
\text { slope } \\
\text { (foot } \\
\text { per mile) }\end{array}$ \\
\hline & $\Gamma$ First Rank Second Rank Third Rank & & & & & & & & & & & & \\
\hline & Fifth $\mathrm{F}$ & & & & & & & & & & & & \\
\hline 1808500 & Grove Lake above outlet to North Fork Crow River & SE SW & 26 & $125 \mathrm{~N}$ & $36 \mathrm{~W}$ & 14.5 & 5.0 & 24.5 & 14.5 & 5.0 & 24.5 & 7.95 & 2.2 \\
\hline 1800100 & North Fork Crow River above Judicial Ditch No. 1 & NW SW & 04 & $124 \mathrm{~N}$ & $35 \mathrm{~W}$ & 14.6 & 0.1 & 14.8 & 29.1 & 2.5 & 19.6 & 12.6 & 4.1 \\
\hline 1800300 & Judicial Ditch No.1 to North Fork Crow River & NW SW & 04 & $124 \mathrm{~N}$ & $35 \mathrm{~W}$ & 14.6 & 0.0 & 11.1 & 14.6 & 0.0 & 11.1 & 9.82 & 5.9 \\
\hline 1807102 & North Fork Crow River above County Ditch No. 32 & SW SE & 15 & $124 \mathrm{~N}$ & $35 \mathrm{~W}$ & 4.56 & 0.0 & 11.4 & 48.3 & 1.5 & 16.3 & 15.8 & 4.9 \\
\hline 1807200 & County Ditch No. 32 to North Fork Crow River & SE SE & 15 & $124 \mathrm{~N}$ & $35 \mathrm{~W}$ & 18.0 & 1.4 & 16.5 & 18.0 & 1.4 & 16.5 & 10.6 & 3.8 \\
\hline 1807101 & North Fork Crow River above Sedan Brook & SE SW & 23 & $124 \mathrm{~N}$ & $35 \mathrm{~W}$ & 1.94 & 0.0 & 12.0 & 68.2 & 1.4 & 16.2 & 17.2 & 4.9 \\
\hline 1800200 & Sedan Brook to North Fork Crow River & SE SW & 23 & $124 \mathrm{~N}$ & $35 \mathrm{~W}$ & 29.0 & 0.0 & 19.2 & 29.0 & 0.0 & 19.2 & 12.6 & 5.3 \\
\hline 1807100 & North Fork Crow River above County Ditch No. 7 & NW SW & 30 & $124 \mathrm{~N}$ & $35 \mathrm{~W}$ & 3.16 & 0.0 & 8.9 & 100. & 1.0 & 16.8 & 19.6 & 4.8 \\
\hline 1806900 & County Ditch No. 7 to North Fork Crow River & NW SW & 30 & $124 \mathrm{~N}$ & $35 \mathrm{~W}$ & 8.37 & 0.0 & 18.3 & 8.37 & 0.0 & 18.3 & 7.00 & 7.1 \\
\hline 1806801 & North Fork Crow River above County Ditch No. 7 & SE SE & 30 & $124 \mathrm{~N}$ & $34 \mathrm{~W}$ & 0.50 & 0.0 & 12.6 & 109. & 0.9 & 16.9 & 20.5 & 4.8 \\
\hline 1807000 & County Ditch No. 7 to North Fork Crow River & SE SE & 30 & $124 \mathrm{~N}$ & $34 \mathrm{~W}$ & 9.93 & 0.0 & 6.1 & 9.93 & 0.0 & 6.1 & 6.30 & 9.3 \\
\hline 1806800 & North Fork Crow River above Skunk River & NW SW & 03 & $123 \mathrm{~N}$ & $34 \mathrm{~W}$ & 10.1 & 0.0 & 13.8 & 129. & 0.8 & 15.8 & 24.0 & 4.6 \\
\hline 1800400 & $\begin{array}{l}\text { Skunk River (County Ditch No. 29) to North Fork } \\
\text { Crow River }\end{array}$ & NW SW & 03 & $123 \mathrm{~N}$ & $34 \mathrm{~W}$ & 33.3 & 1.2 & 20.8 & 33.3 & 1.2 & 20.8 & 18.6 & 5.0 \\
\hline 1806601 & $\begin{array}{l}\text { North Fork Crow River above gaging station near } \\
\text { Georgeville: station number is } \mathbf{0 5 2 7 5 9 7 0}\end{array}$ & SE SE & 03 & $123 \mathrm{~N}$ & $34 \mathrm{~W}$ & 2.17 & 0.0 & 8.6 & 165. & 0.8 & 16.7 & 25.4 & 4.7 \\
\hline 1806700 & Unnamed tributary to North Fork Crow River & NE SE & 25 & $123 \mathrm{~N}$ & $34 \mathrm{~W}$ & 8.89 & 5.5 & 16.1 & 8.89 & 5.5 & 16.1 & 8.46 & 5.4 \\
\hline 1806600 & $\begin{array}{l}\text { North Fork Crow River above unnamed tributary } \\
\text { subbasin } 1806500\end{array}$ & NW SE & 25 & $123 \mathrm{~N}$ & $34 \mathrm{~W}$ & 16.5 & 0.0 & 16.9 & 190. & 1.0 & 16.7 & 31.4 & 4.6 \\
\hline 1806500 & Unnamed tributary to North Fork Crow River & NW SE & 25 & $123 \mathrm{~N}$ & $34 \mathrm{~W}$ & 6.09 & 0.0 & 3.7 & 6.09 & 0.0 & 3.7 & 7.55 & 7.7 \\
\hline 1803701 & $\begin{array}{l}\text { North Fork Crow River above gaging station near } \\
\text { Regal: station number is } \mathbf{0 5 2 7 6 0 0 0}\end{array}$ & SE SW & 11 & $122 \mathrm{~N}$ & $33 \mathrm{~W}$ & 16.6 & 0.0 & 22.7 & 213. & 0.9 & 16.8 & 39.9 & 4.2 \\
\hline
\end{tabular}


Table 1.-Physical characteristic data for the North Fork Crow-Crow River Basin-Continued.

\begin{tabular}{|c|c|c|c|c|c|c|c|c|c|c|c|c|c|}
\hline \multirow[b]{2}{*}{$\begin{array}{l}\text { Basin } \\
\text { number }\end{array}$} & \multirow[b]{2}{*}{ Stream name and location } & \multicolumn{4}{|c|}{ Outlet location } & \multicolumn{3}{|c|}{ By subbasin } & \multicolumn{5}{|c|}{ Cumulative to mouth of basin } \\
\hline & & $\begin{array}{l}\text { Quarter- } \\
\text { quarter } \\
\text { section }\end{array}$ & Section & $\begin{array}{l}\text { Town- } \\
\text { ship }\end{array}$ & Range & $\begin{array}{l}\text { Drainage } \\
\text { area } \\
\text { (square } \\
\text { miles) }\end{array}$ & $\begin{array}{c}\text { Lake } \\
\text { area } \\
\text { (percent } \\
\text { of } \\
\text { subbasin } \\
\text { area) }\end{array}$ & $\begin{array}{c}\text { Storage } \\
\text { area } \\
\text { (percent } \\
\text { of } \\
\text { subbasin } \\
\text { area) }\end{array}$ & $\begin{array}{l}\text { Drainage } \\
\text { area } \\
\text { (square } \\
\text { miles) }\end{array}$ & $\begin{array}{l}\text { Lake } \\
\text { area } \\
\text { (percent } \\
\text { of total } \\
\text { area) }\end{array}$ & $\begin{array}{l}\text { Storage } \\
\text { area } \\
\text { (percent } \\
\text { of total } \\
\text { area) }\end{array}$ & $\begin{array}{l}\text { Main } \\
\text { channel } \\
\text { length } \\
\text { (miles) }\end{array}$ & $\begin{array}{c}\text { Main } \\
\text { channel } \\
\text { slope } \\
\text { (foot } \\
\text { per mile) }\end{array}$ \\
\hline & First Rank Second Rank Third Rank & & & & & & & & & & & & \\
\hline 1803700 & $\begin{array}{l}\text { North Fork Crow River above unnamed tributary } \\
\text { subbasin } 1803600\end{array}$ & SE NE & 14 & $122 \mathrm{~N}$ & $33 \mathrm{~W}$ & 0.17 & 0.0 & 34.4 & 213. & 0.9 & 16.8 & 40.8 & 4.1 \\
\hline 1803600 & Unnamed tributary to North Fork Crow River & SE NE & 14 & $122 \mathrm{~N}$ & $33 \mathrm{~W}$ & 15.6 & 0.1 & 22.5 & 15.6 & 0.1 & 22.5 & 8.58 & 5.8 \\
\hline 1804304 & $\begin{array}{l}\text { Unnamed tributary to North Fork Crow River above } \\
\text { gaging station near Paynesville: station number is } \\
\mathbf{0 5 2 7 6 1 0 0}\end{array}$ & SW NW & 12 & $122 \mathrm{~N}$ & $33 \mathrm{~W}$ & 0.49 & 0.0 & 8.4 & 0.49 & 0.0 & 8.4 & 1.11 & 44.4 \\
\hline 1804303 & $\begin{array}{l}\text { North Fork Crow River above gaging station at } \\
\text { Paynesville: station number is } \mathbf{0 5 2 7 6 2 0 0}\end{array}$ & SE SW & 09 & $122 \mathrm{~N}$ & $32 \mathrm{~W}$ & 14.0 & 0.3 & 11.9 & 243. & 0.8 & 16.9 & 46.3 & 4.5 \\
\hline 1804302 & $\begin{array}{l}\text { North Fork Crow River above unnamed tributary } \\
\text { (County Ditch No. 5) }\end{array}$ & NE SE & 09 & $122 \mathrm{~N}$ & $32 \mathrm{~W}$ & 0.41 & 0.0 & 2.7 & 244. & 0.8 & 16.9 & 47.4 & 4.6 \\
\hline 1804200 & $\begin{array}{l}\text { Unnamed tributary (County Ditch No. 5) to North } \\
\text { Fork Crow River }\end{array}$ & NE SE & 09 & $122 \mathrm{~N}$ & $32 \mathrm{~W}$ & 9.67 & 0.0 & 11.2 & 9.67 & 0.0 & 11.2 & 11.3 & 12.7 \\
\hline 1804301 & $\begin{array}{l}\text { North Fork Crow River above gaging station at } \\
\text { Paynesville: station number is } \mathbf{0 5 2 7 6 2 1 0}\end{array}$ & SE SW & 10 & $122 \mathrm{~N}$ & $32 \mathrm{~W}$ & 0.47 & 0.2 & 10.4 & 254. & 0.8 & 16.6 & 47.4 & 4.6 \\
\hline 1804300 & North Fork Crow River to Rice Lake & SE NE & 24 & $122 \mathrm{~N}$ & $32 \mathrm{~W}$ & 4.93 & 0.0 & 23.7 & 259. & 0.8 & 16.8 & 51.6 & 4.8 \\
\hline 1806400 & Rice Lake outlet to North Fork Crow River & NE SE & 24 & $122 \mathrm{~N}$ & $32 \mathrm{~W}$ & 16.1 & 15.3 & 25.8 & 16.1 & 15.3 & 25.8 & 6.12 & 11.8 \\
\hline 1802500 & Unnamed tributary to Lake Koronis & NE SW & 31 & $122 \mathrm{~N}$ & $32 \mathrm{~W}$ & 6.88 & 0.0 & 16.7 & 6.88 & 0.0 & 16.7 & 4.48 & 25.0 \\
\hline 1802700 & Unnamed tributary to Lake Koronis & SW NW & 03 & $121 \mathrm{~N}$ & $32 \mathrm{~W}$ & 10.2 & 0.0 & 17.8 & 10.2 & 0.0 & 17.8 & 7.76 & 12.3 \\
\hline 1802600 & $\begin{array}{l}\text { Koronis Lake outlet above gaging station near } \\
\text { Paynesville: station number is } \mathbf{0 5 2 7 6 3 0 0}\end{array}$ & SW SE & 03 & $121 \mathrm{~N}$ & $32 \mathrm{~W}$ & 19.5 & 24.9 & 36.2 & 311. & 3.0 & 18.5 & 57.3 & 4.5 \\
\hline 1805501 & $\begin{array}{l}\text { North Fork Crow River above Unnamed tributary } \\
\text { subbasin } 1805600\end{array}$ & SE SE & 14 & $121 \mathrm{~N}$ & $32 \mathrm{~W}$ & 4.19 & 2.2 & 18.6 & 316. & 3.0 & 18.5 & 60.5 & 4.2 \\
\hline 1805600 & Unnamed ditch to North fork Crow River & SE SE & 14 & $121 \mathrm{~N}$ & $31 \mathrm{~W}$ & 6.98 & 1.7 & 19.7 & 6.98 & 1.7 & 19.7 & 6.25 & 15.4 \\
\hline
\end{tabular}


Table 1.-Physical characteristic data for the North Fork Crow-Crow River Basin-Continued.

\begin{tabular}{|c|c|c|c|c|c|c|c|c|c|c|c|c|c|}
\hline \multirow[b]{2}{*}{$\begin{array}{c}\text { Basin } \\
\text { number }\end{array}$} & \multirow[b]{2}{*}{ Stream name and location } & \multicolumn{4}{|c|}{ Outlet location } & \multicolumn{3}{|c|}{ By subbasin } & \multicolumn{5}{|c|}{ Cumulative to mouth of basin } \\
\hline & & $\begin{array}{l}\text { Quarter- } \\
\text { quarter } \\
\text { section }\end{array}$ & Section & $\begin{array}{l}\text { Town- } \\
\text { ship }\end{array}$ & Range & $\begin{array}{c}\text { Drainage } \\
\text { area } \\
\text { (square } \\
\text { miles) }\end{array}$ & $\begin{array}{c}\text { Lake } \\
\text { area } \\
\text { (percent } \\
\text { of } \\
\text { subbasin } \\
\text { area) }\end{array}$ & $\begin{array}{c}\text { Storage } \\
\text { area } \\
\text { (percent } \\
\text { of } \\
\text { subbasin } \\
\text { area) }\end{array}$ & $\begin{array}{c}\text { Drainage } \\
\text { area } \\
\text { (square } \\
\text { miles) }\end{array}$ & $\begin{array}{l}\text { Lake } \\
\text { area } \\
\text { (percent } \\
\text { of total } \\
\text { area) }\end{array}$ & $\begin{array}{l}\text { Storage } \\
\text { area } \\
\text { (percent } \\
\text { of total } \\
\text { area) }\end{array}$ & $\begin{array}{l}\text { Main } \\
\text { channel } \\
\text { length } \\
\text { (miles) }\end{array}$ & $\begin{array}{c}\text { Main } \\
\text { channel } \\
\text { slope } \\
\text { (foot } \\
\text { per mile) }\end{array}$ \\
\hline 1805500 & North Fork Crow River above Middle Fork Crow River & $\mathrm{NEN}$ & 32 & $121 \mathrm{~N}$ & 31W & 8.00 & 00 & 170 & 330 & 29 & 185 & 660 & 30 \\
\hline 1803800 & County Ditch No. B6 to Middle Fork Crow River & NE NW & 08 & $122 \mathrm{~N}$ & $34 \mathrm{~W}$ & 21.1 & 2.9 & 26.4 & 21.1 & 2.9 & 26.4 & 11.8 & 6.1 \\
\hline 1804500 & $\begin{array}{l}\text { Middle Fork Crow River above County Ditch No. } \\
\text { B6 }\end{array}$ & NE NW & 08 & $122 \mathrm{~N}$ & $34 \mathrm{~W}$ & 15.8 & 0.0 & 16.1 & 15.8 & 0.0 & 16.1 & 8.31 & 5.8 \\
\hline 1802400 & County Ditch No. 37 to Middle Fork Crow River & NE NW & 28 & $122 \mathrm{~N}$ & $34 \mathrm{~W}$ & 23.6 & 1.6 & 27.6 & 23.6 & 1.6 & 27.6 & 11.8 & 6.9 \\
\hline 1803901 & Middle Fork Crow River above County Ditch No. 37 & SE SW & 21 & $122 \mathrm{~N}$ & $34 \mathrm{~W}$ & 11.1 & 0.0 & 32.6 & 71.6 & 1.4 & 25.5 & 16.6 & 5.4 \\
\hline 1803900 & Middle Fork Crow River to Mud Lake & NE SE & 28 & $122 \mathrm{~N}$ & $34 \mathrm{~W}$ & 0.45 & 0.0 & 59.0 & 72.1 & 1.4 & 25.7 & 17.6 & 5.1 \\
\hline 1803500 & Unnamed tributary to Middle Fork Crow River & SE SE & 35 & $122 \mathrm{~N}$ & $34 \mathrm{~W}$ & 6.55 & 8.3 & 17.9 & 6.55 & 8.3 & 17.9 & 4.08 & 1.4 \\
\hline 1804000 & $\begin{array}{l}\text { Middle Fork Crow River above gaging station at New } \\
\text { London: station number is } \mathbf{0 5 2 7 7 0 0 0}\end{array}$ & NE SW & 10 & $121 \mathrm{~N}$ & $34 \mathrm{~W}$ & 21.4 & 12.9 & 35.6 & 100. & 4.3 & 27.3 & 22.9 & 3.8 \\
\hline 1802300 & Middle Fork Crow River above Nest Lake inlet & SW SW & 21 & $121 N$ & $34 \mathrm{~W}$ & 12.2 & 2.3 & 27.3 & 112. & 4.1 & 27.3 & 30.1 & 4.0 \\
\hline 1802200 & Nest Lake outlet to Green Lake & NW NE & 27 & $121 \mathrm{~N}$ & $34 \mathrm{~W}$ & 9.55 & 20.4 & 38.7 & 122. & 5.4 & 28.2 & 30.1 & 4.0 \\
\hline 1804100 & $\begin{array}{l}\text { Green Lake outlet above gaging station near Spicer: } \\
\text { station number is } \mathbf{0 5 2 7 7 3 0 0}\end{array}$ & NE NE & 30 & $121 \mathrm{~N}$ & $33 \mathrm{~W}$ & 23.8 & 40.9 & 48.1 & 146. & 11.2 & 31.5 & 33.3 & 4.1 \\
\hline 1803400 & $\begin{array}{l}\text { Lake Calhoun outlet above gaging station near } \\
\text { Spicer: station number is } \mathbf{0 5 2 7 7 6 0 0}\end{array}$ & SW NE & 28 & $121 \mathrm{~N}$ & $33 \mathrm{~W}$ & 12.0 & 8.5 & 23.1 & 12.0 & 8.5 & 23.1 & 6.11 & 12.2 \\
\hline 1803301 & $\begin{array}{l}\text { Middle Fork Crow River above gaging station near } \\
\text { Spicer: station number is } \mathbf{0 5 2 7 8 0 0 0}\end{array}$ & SE NE & 27 & $121 \mathrm{~N}$ & $33 \mathrm{~W}$ & 5.83 & 1.0 & 28.9 & 163. & 10.6 & 30.7 & 36.6 & 3.6 \\
\hline 1803300 & $\begin{array}{l}\text { Middle Fork Crow River above unnamed tributary } \\
\text { subbasin } 1803200\end{array}$ & NW SW & 36 & $121 \mathrm{~N}$ & $33 \mathrm{~W}$ & 8.75 & 0.0 & 22.0 & 172. & 10.1 & 30.3 & 38.4 & 3.5 \\
\hline 1803100 & Unnamed tributary to Diamond Lake & NE SE & 28 & $120 \mathrm{~N}$ & $33 \mathrm{~W}$ & 13.6 & 7.3 & 15.6 & 13.6 & 7.3 & 15.6 & 4.82 & 12.6 \\
\hline 1803200 & Unnamed tributary to Middle Fork Crow River & NW SW & 36 & $121 \mathrm{~N}$ & $33 \mathrm{~W}$ & 24.6 & 11.2 & 21.8 & 38.2 & 9.8 & 19.6 & 12.7 & 4.1 \\
\hline 1802800 & Middle Fork Crow River above County Ditch No. 47 & NW SW & 32 & $121 \mathrm{~N}$ & $32 \mathrm{~W}$ & 5.75 & 0.0 & 11.0 & 216. & 9.7 & 27.9 & 40.5 & 3.3 \\
\hline
\end{tabular}


Table 1.-Physical characteristic data for the North Fork Crow-Crow River Basin-Continued.

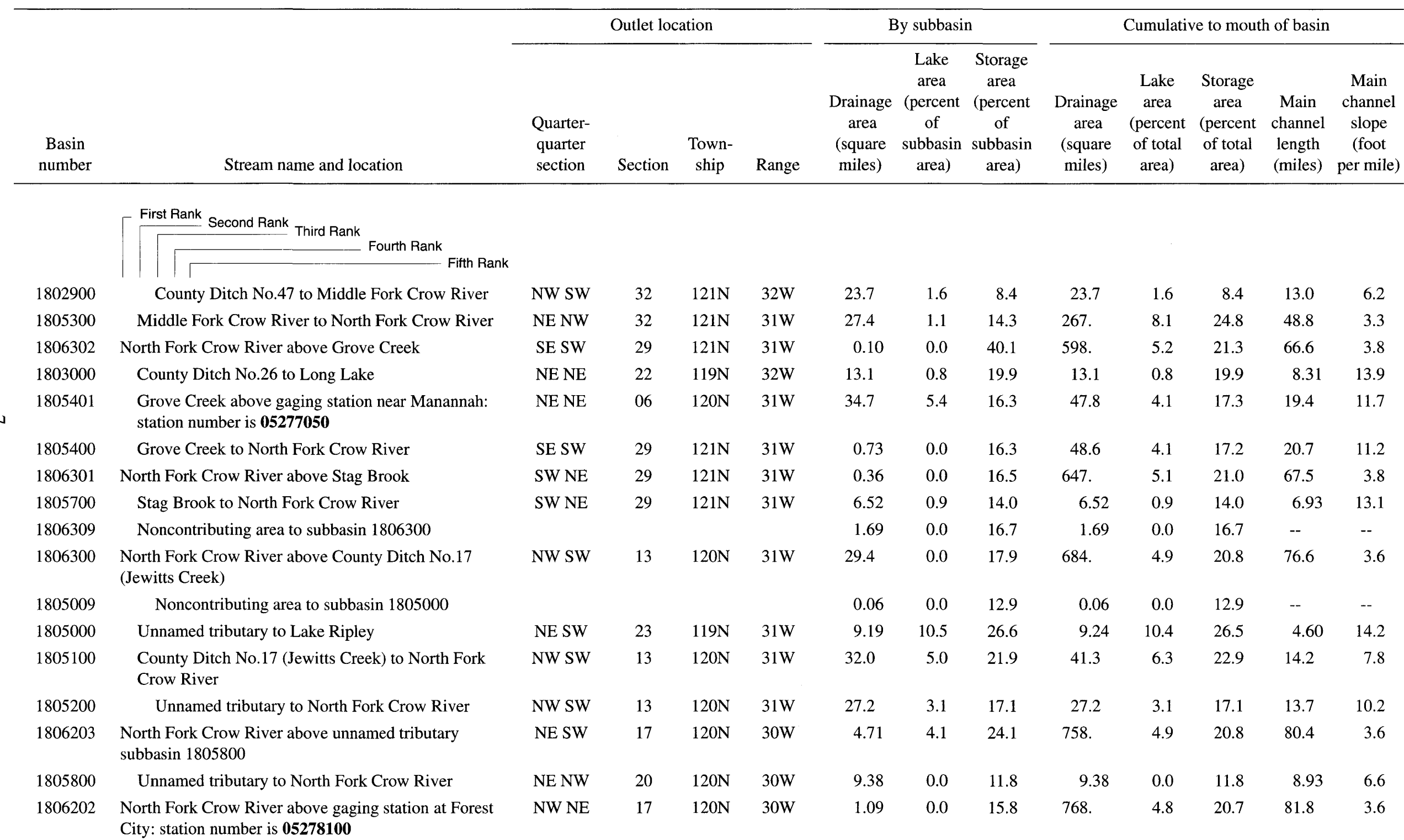


Table 1.-Physical characteristic data for the North Fork Crow-Crow River Basin-Continued.

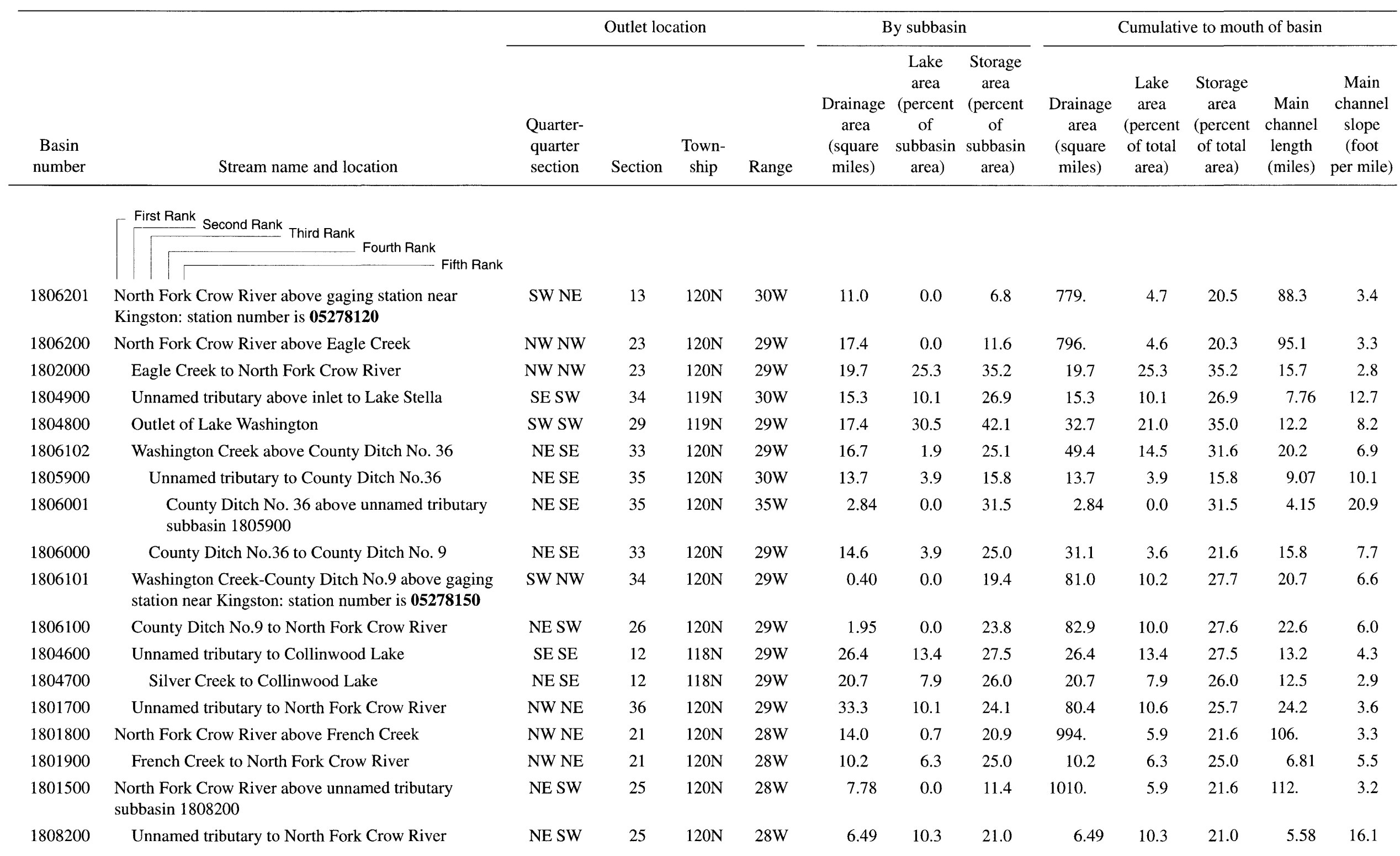


Table 1.-Physical characteristic data for the North Fork Crow-Crow River Basin-Continued.

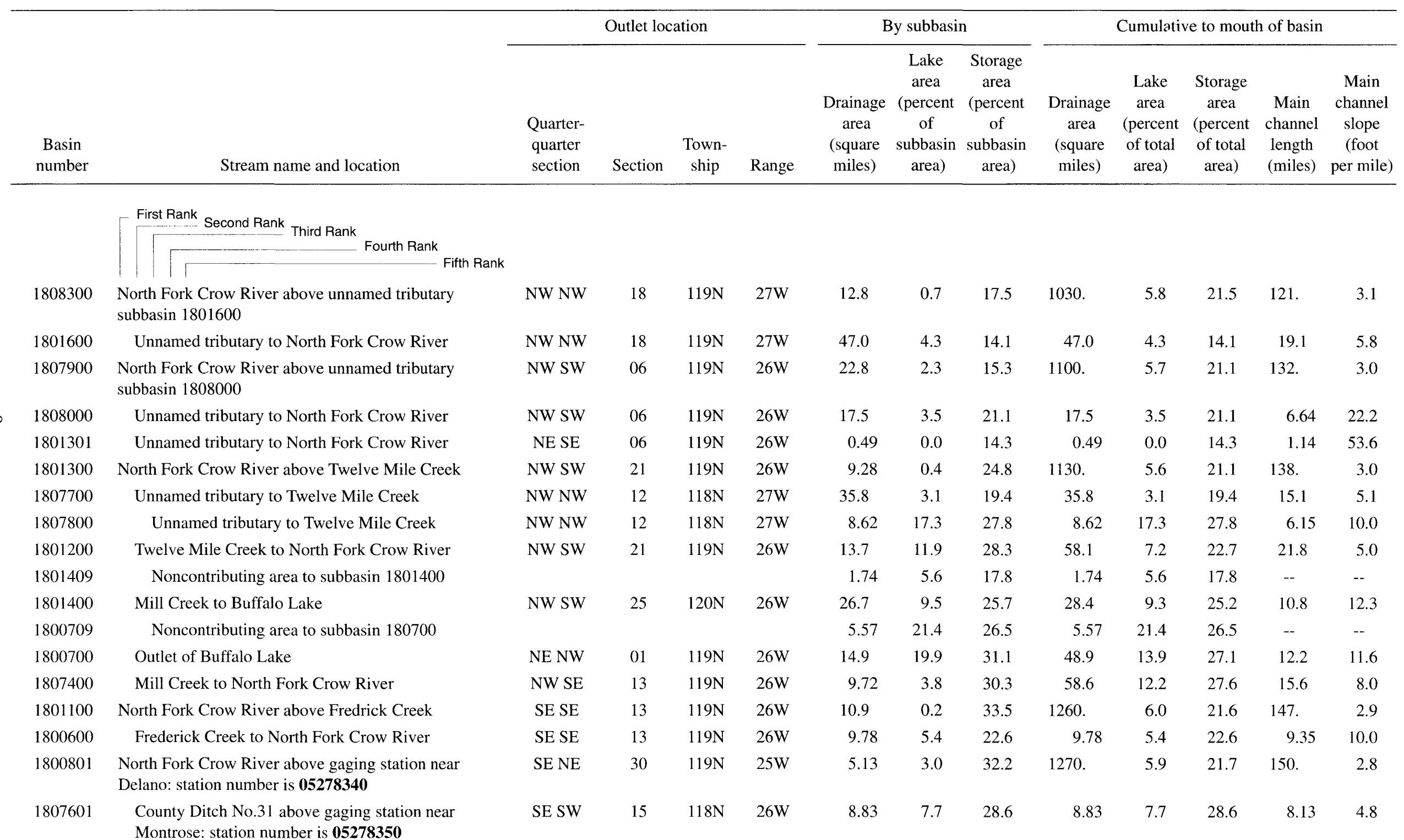


Table 1.-Physical characteristic data for the North Fork Crow-Crow River Basin-Continued.

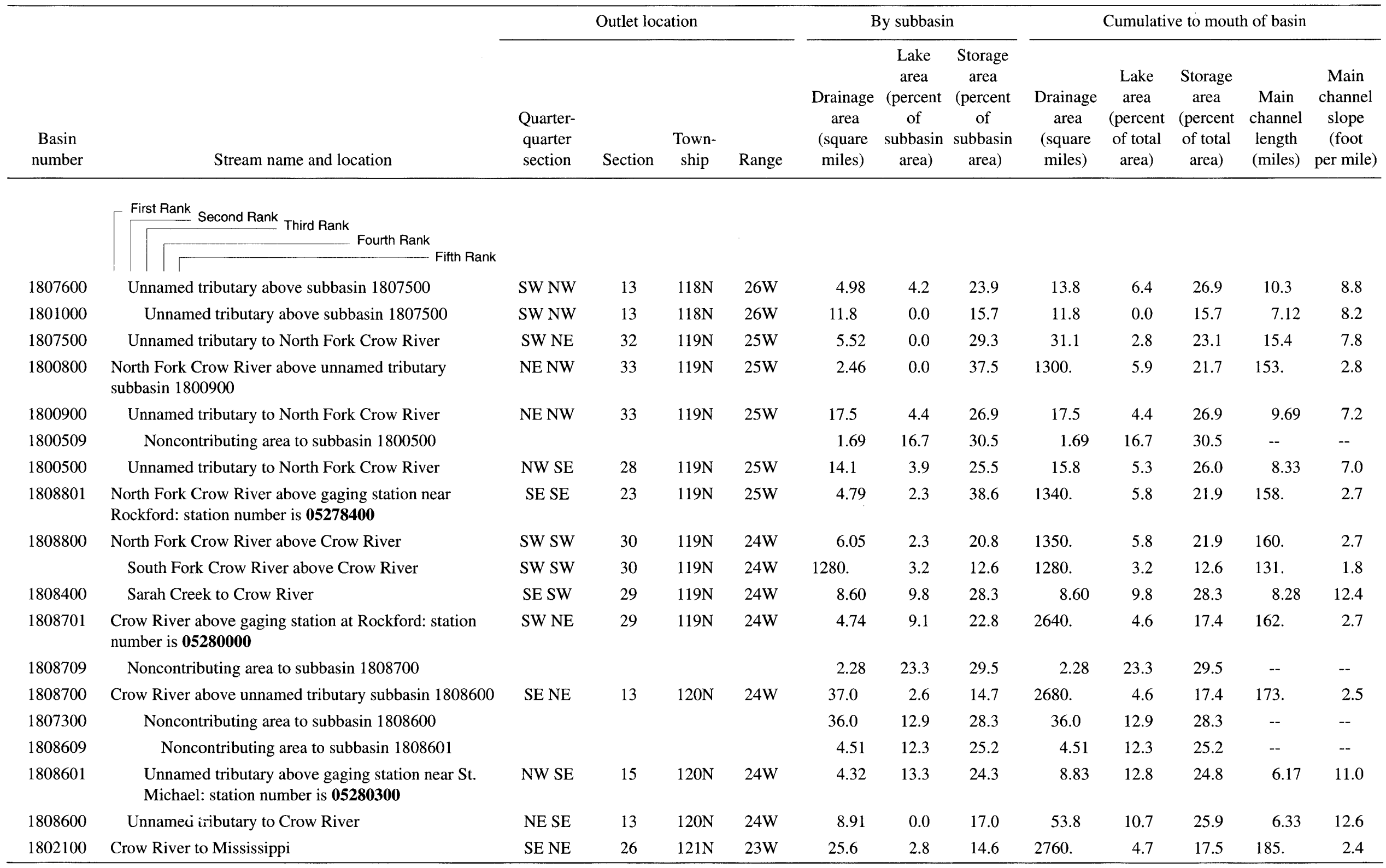

